\section{Compression-Decompression (ACD)-CPR}

T.S. Mutzbauer; W. Stabl; K.H. Lindner

Department of Anesthesiology and Critical Care Medicine, University of Ulm, Ulm, Germany

Objective: Different studies have demonstrated the respiratory effects associated with the use of the cardiopump during ACD-CPR in animal models. There is no study, however, demonstrating beneficial ventilatory effects of this tool in humans with cardiac arrest. The aim of this evaluation was to measure tidal volumes applied by the cardio-pump as used by medical personnel experienced in conventional cardiopulmonary resuscitation (CCPR).

Materials and Methods: A 35 year-old normothermic male with a large posterior myocardial infarction underwent CCPR (38 minutes) followed by ACD-CPR (50 minutes) due to cardiac arrest. As resuscitative efforts remained unsuccessful, termination of the resuscitation was decided (total dose: $60 \mathrm{mg}$ epinephrine). Minute volumes applied by the emergency physician and three EMTs during ACD-CPR were measured using a Draeger Oxylog 2000 in the CPAP mode (CPAP = 0), and the frequency of chest compressions was assessed using a Nellcor pulse oximeter. Shortly after the test, the patient showed return of spontaneous circulation (ROSC) and was transferred to the ICU. 50 minutes after ROSC, CCPR was continued for 30 minutes due to refractory pulseless electrical activity. Immediately after decision to stop CPR, two medical intensive care physicians and one ICU nurse continued with ACDCPR. Minute volumes were measured with a Draeger Anemone volumeter. Additionally, arterial blood pressure during CCPR and ACD-CPR were recorded by a Siemens Sirecust 1281 Monitor via femoral artery catheter. All "rescuers" were blinded with respect to the measured parameters.

Results: The calculated median tidal volume was $143 \mathrm{ml}$ (range 88 to $200 \mathrm{ml}$ ). Mean arterial blood pressures (BP) were 40 vs. 50.4 vs. 38.4 vs. $36 \mathrm{mmHg}$ with CCPR vs. ACD-CPR.

$\begin{array}{lcccc} & \begin{array}{c}\text { Minute } \\ \text { Ventilation }\end{array} & \begin{array}{c}\text { Tidal } \\ \text { Volume } \\ \text { [1/min] }\end{array} & \text { BP ACD } & \text { BP CCPR } \\ \text { [mmHg] } & \text { [mmHg] } \\ \text { EM physician } & 10 & 143 & & \\ \text { EMT1 } & 7 & 88 & & \\ \text { EMT2 } & 10 & 100 & & \\ \text { EMT3 } & 8 & 80 & & \\ \text { ICU physician 1 } & 7.6 & 200 & 70 / 40 & 60 / 30 \\ \text { ICU physician 2 } & 9.6 & 160 & 49 / 30 & 60 / 36 \\ \text { ICU nurse } & 8 & 200 & 47 / 34 & 60 / 32\end{array}$

Discussion: Results indicate that at least a "median frequency ventilation" could be achieved by ACD-CPR. Some patients might benefit from ACD-CPR without additional ventilation, because ACD-CPR could induce sufficient brain perfusion and subsequently spontaneous ventilation leading to even larger tidal volumes, active compression-decompression CPR, ventilation, tidal volumes, and median frequency ventilation.

Key Words: active compression-decompression; car- diopulmonary resuscitation (CPR); ventilation

\section{Prehospital Thoracotomy for Cardiac Arrest Due to Perforating Chest Wounds: Case Reports of Two Patients \\ T. Silfuast \\ Department of Anaesthesia, Helsinki University Hospital, Helsinki, Finland}

Prehospital cardiac arrest due to trauma almost uniformly is fatal. In penetrating chest trauma, survival may be possible if emergency thoracotomy is rapidly performed. Two patients are described for whom emergency thoracotomy was performed at the site of the stabbing.

Patient 1, a 27-year-old male, was found unresponsive by the responding physician who staffed prehospital emergency unit (PECU), but the victim still had agonal breathing No pulses were palpable and the ECG showed pulseless electrical activity (PEA). Three stab wounds were found anteriorly in the chest, and two more in the neck. After intubation, a left thoracotomy was performed. The distended pericardium was opened, and the heart started beating when direct compressions were started. The patient then was transported to the hospital. In the operating room $(\mathrm{OR})$, the left ventricle was found to be perforated antero-posteriorly. After suturing, profuse bleeding from the neck continued. The patient never achieved adequate blood pressure and died in the operating room.

Patient 2, a 24-year-old male, had one stab wound to the left of the sternum. On the arrival of the PECU, he suddenly became lifeless and the ECG showed PEA. Transportation time to hospital was estimated to be 18 minutes. A left thoracotomy was performed after intubation, and at pericardiotomy, a clot was removed and the heart spontaneously resumed beating. The patient was transported while the physician obstructed the cardiac wound with his finger. In the OR, the wound was sutured, and the patient subsequently made a complete recovery and was discharged to home intact.

Conclusion: It is concluded that prehospital thoracotomy may be lifesaving in selected trauma cases, especially if the lesions are expected to be few in number.

Key Words: cardiac arrest; prehospital; stabbing; thoracotomy; trauma

\section{Evaluation of Nation-wide Education of Basic Life Support (BLS) in Japan} M. Aono, MD; S. Kawaguchi; MD; R. Tamai, RN, ELST; A. Taknabe, RN, ELST

Kanazawa Medical University, Uchinada Ishikawa-Ken, Japan

Introduction: In Japan, since 1994, all persons who would wish to acquire a driver's license at a driving school must participate in at least two hours of training in Basic Life Support (BLS). More than 250 million people get a driver's license every year which almost equals $2 \%$ of the total population of Japan. Therefore, the teaching of BLS and especially its evaluation, is quite important. 